\title{
ENHANCED NESTED VIDEO WATERMARKING USING WAVELETS AND GEOMETRIC WARPING
}

\author{
T.Jayamalar ${ }^{1}$ and Dr. V. Radha ${ }^{2}$ \\ ${ }^{1}$ Research Scholar, Avinashilingam Deemed University for Women, Coimbatore, India. \\ jayamalarskjin@gmail.com \\ ${ }^{2}$ Associate Professor, Avinashilingam Deemed University for Women, Coimbatore, \\ India. \\ radharesearcheyahoo.com
}

\begin{abstract}
The amount of information created in the current era of information explosion is increasing in a overwhelming fashion. This increase in content has increased the awareness to protect the intellectual property. This paper presents one such method to protect Video content. The proposed watermarking hybrid scheme combines visual cryptography and wavelets to create a nested watermark, which is embedded into the raw uncompressed video signal using geometric warping technology. A block selection algorithm is used to select relevant area for embedding so as to improve robustness. The experimental results showed that the proposed system shows significant improvement in terms of capacity and robustness against various attacks.
\end{abstract}

\section{KEYWORDS}

Video Watermarking, Nested Watermarking, Geometric Warping, Wavelets.

\section{INTRODUCTION}

Digital Watermarking is a data hiding technique where an information or message is hidden inside a signal transparent to the user. It deals with embedding information like author name, status and recipient into the host data in such a way that it remains transparent or undetectable. The watermark information should be embedded in such a way that it is not detectable or removable even after many spurious or innocuous attempts. Watermarking is applicable to many forms of digital data, like text, image, audio and video where copyright needs to be protected. Among the various techniques available, digital watermarking techniques for video have gained more attention in proving the integrity and authenticity of the owner ([25], [17], [18]).

A watermark can be text, image ([19], [7], [9], [14]), video ([5],[10]), audio or three dimensional data [26]. Among them image and video watermarking are two important areas that has attracted several researchers ([33], [21]). Methods for embedding watermark information may vary between types of media, but the basis of these methods remain more or less same. Video watermarking applications include copy control, fingerprinting, ownership identification, authentication, video tagging, digital video broadcast monitoring and media bridge. 
The International Journal of Multimedia \& Its Applications (IJMA) Vol.3, No.4, November 2011

In video watermarking, it works with the principle that they should ensure digital ownership, have the ability to track the source of the digital video, have quick and easy way to detect video manipulation, have no visible video degradation and should have easy implementation procedure. Video watermarking is considered challenging because video sequences consists of a series of consecutive and equally time spaced still images which (i) require Larger Space (ii) have realtime constraints, (iii) are in compressed domain and (iv) are susceptible to attacks such as frame averaging, frame dropping, frame swapping etc. The basic characteristics are imperceptibility, reliability, low complexity of watermarking algorithm and security of the hiding place.

In general scenario, watermarks are embedded into video signal either during or after compression ([29], 28]). This has the advantage that watermarking process is not influenced by the compression algorithm. However, the watermarks are generally not robust to a transcoding of the video. Thus, embedding the watermark to the uncompressed video data will have the advantage of being robust to the transcoding of the video. The challenge here is that since both watermarking technique and compression algorithm both seek to use irrelevant data, the embedded watermark might be removed by the compression algorithm. It would be better if the watermark is embedded in the relevant data so that it is not removed by compression algorithm. A method based on geometric warping was suggested by [27]. This method was efficient in terms robustness to lossy compression and improved the quality by using a block linking approach. This method had the advantage of not requiring the original video while extracting the watermark. However, this technique reduced the watermark capacity. This method is referred as base system in this paper. Aggarwal et al. [1] proposed another efficient watermarking algorithm to improve the payload and robustness without affecting the image quality. They used a concept of nested watermarking where one watermark is embedding into another watermark and the resultant image is embedded into the cover watermark. The concept of nested watermark improved payload and was able to maintain the quality to a great extent.

Motivated by these two works, in the present work, a single copyright message is divided into two shares using Visual Cryptography (VC). The second share is then embedded into first share, thus creating a new watermark image. This image is then embedded into an uncompressed video signal using geometric warping method as suggested by [27].

The rest of the paper is organized as follows. Section 2 presents a brief study on the previous works related to the topic. In Section 3, the basic concepts of visual cryptography (VC), wavelets and geometric warping are described. Section 4 explains the proposed watermarking algorithm. Section 5 presents the experimental results and Section 6 concludes the work with future research directions.

\section{Previous STUdies}

Solutions to video watermarking have been studied by various researchers who work with both raw uncompressed video data [8] and compressed video data [2]. Video watermarking techniques can be mainly grouped as spatial domain approaches and frequency domain approaches.

The spatial domain watermarking techniques embed the watermark by modifying the pixel values of the host image directly. Least Significant bit (LSB) technique is the most frequently used method [16]. Both [30] and [13] embedded the watermark in saturation on the HIS (Hue, Saturation, Intensity) color space. A variable block size based adaptive watermarking in spatial domain was proposed by [15]. In a later period, [32] proposed a probability block based watermarking method for color image with fixed block size. Recently, an algorithm based on chaotic maps was proposed by [34] to determine the pixel bit for embedding. The main advantages of pixel based methods are that they are conceptually simple and have very low 
The International Journal of Multimedia \& Its Applications (IJMA) Vol.3, No.4, November 2011

computational complexities. However, they also exhibit some major limitations. The need for absolute spatial synchronization leads to high susceptibility to de-synchronization attacks; lack of consideration of the temporal axis results in vulnerability to video processing and multiple frame collusion; and watermark optimization is difficult using only spatial analysis techniques.

Frequency domain approaches use transformation techniques to embed the watermark into a video sequence. Discrete Cosine Transformation (DCT), Discrete Fourier Transformation (DFT) and Discrete Wavelet Transformation (DWT) are the three main methods of data transformation. A subsample based watermarking technique was proposed by [20], where the DCT coefficients of the subimages were utilized to store the watermark. The authors of [6], proposed an algorithm which was based on embedding the watermark image three times at three different frequency bands, namely, low, medium and high and the results proved that the watermark cannot be totally destroyed by either low pass, medium or high pass filter. In [29], two complementary watermarks were embedded into the host image in order to make it difficult for attackers to destroy both of them. The main benefit obtained from frequency domain techniques is that they can take advantage of properties of alternate domains to address the limitations of pixel-based methods. Besides, analysis of the host signal in a frequency domain is a prerequisite for applying more advanced masking properties of the HVS to enhance watermark robustness and imperceptibility. Generally, the main drawback of transform domain methods is the high computational requirement.

Another field where scientists focus is on embedding into a compressed domain which can be done during or after the encoding process ([27],[28]). Alternatively, techniques to work on raw format have also received attention. In general, invisible watermark is embedded into irrelevant information of video data which can not be perceived by human eyes. This results in problems because compression algorithms, which also try to remove irrelevant information, might remove watermark accidentally. In [27], this problem was solved by embedding watermark in the relevant information of videos in an imperceptible manner. This approach is based on geometric warping of blocks. This approach though very efficient produced visible artifacts and video quality after extraction was low. Method to prevent these artifacts and improve the video quality was proposed by [27]. They used geometric warping method to embed watermarks, which did not require the original video during watermark extraction. This method improved [23] by removing artifacts and video quality, but was slow for applications where time was significant. This paper proposes a solution to solve this problem.

\section{METHODS USED}

The concepts behind the various methods used in the proposed watermarking scheme are explained in this section.

\subsection{Visual Cryptography}

Visual Cryptography (VC), a paradigm introduced by [22], was initially used to encrypt material like written text, printed text, pictures, in a secure manner. It is a Visual Secret Sharing Scheme (VSSS) which uses the Human Visual System (HVS) to decrypt a secret message without expensive and complicated decoding process [31]. The basic VC system starts with the encoding phase, where a secret image is divided into a collection of ' $m$ ' black and ' $n$ ' white pixels. Each collection of $\mathrm{m} \times \mathrm{n}$ pixels is referred to as a share, which will resemble a noisy image when separately. During decoding phase, these shares or subset of shares are stacked together which will allow the visual recovery of the secret message. It has been applied to many applications, including but not restricted to E-voting system [24], financial documents [11], information hiding 
The International Journal of Multimedia \& Its Applications (IJMA) Vol.3, No.4, November 2011

[4], general access structures [3], visual authentication and identification [23]. More detailed information about visual cryptography can be found in [35].

Out of the many VC schemes proposed, the present work considers the $(2,2)$ VSS scheme proposed by [22]. In this algorithm, each pixel of the copyright image is expanded into $2 * 2$ pixels. To share a white pixel of the secret image, one row from the first 6 rows of table 1 is chosen randomly. Similarly, the two shares of a black pixel are determined by a random selection from the 6 last rows. As a result, an $M^{*} N$ pixels secret image is expanded into two $2 M^{*} 2 N$ pixels share-images.

Considering security of the method, presence of only one share image reveals nothing about the corresponding secret image, i.e., each $2 * 2$ pixels block of one share-image may correspond to either a white pixel or a black pixel of the secret image. As Fig. 1 shows, stacking the shares of a black secret pixel results in 4 black subpixels, whereas only 2 black subpixels is gained by stacking shares of a white secret pixel [12]. So, secret image is revealed to human eyes by stacking the shares without performing any cryptographical computations. An example of VC scheme is shown in Fig.2.

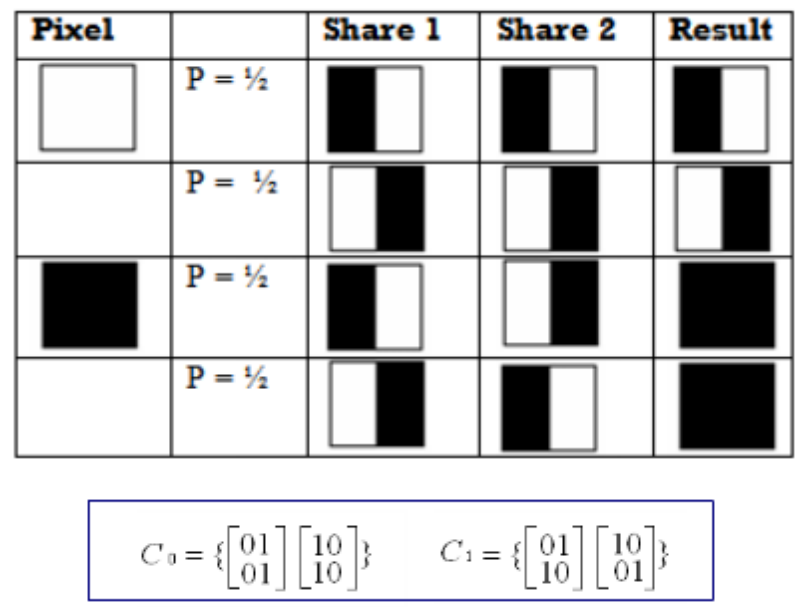

Fig. 1. $(2,2)$ VC Scheme (2 Subpixels)

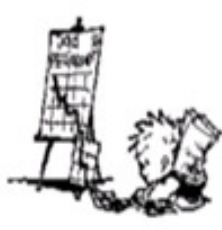

Original Image

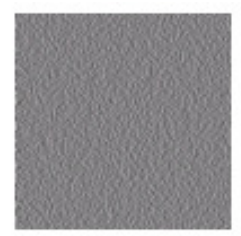

Share 1
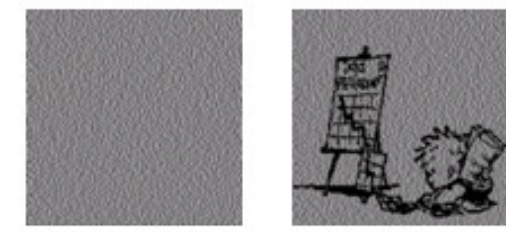

Result image

Fig. .2 Example of VC Scheme

The scheme explained here is used in the proposed system and has the following advantages.

- Simple to implement

- Encryption do not required any heavy computations

- Decryption algorithm not required (uses only Human Visual System).

- Even infinite computation power can not predict the secret message. 
The International Journal of Multimedia \& Its Applications (IJMA) Vol.3, No.4, November 2011

\subsection{Wavelets}

A technique that is frequently used in various domains is the wavelet transformation. Many applications such as compression, signal analysis and processing, watermarking have used wavelets successfully. This section discusses the basic concepts behind Discrete Wavelet Transform (DWT).

In DWT, a signal is segmented into two quadrants, high frequency quadrant (edge details) and low frequency quadrant (other details). The low frequency part is split again into two parts of high and low frequencies and this process is repeated till the signal has been entirely decomposed. In watermarking, generally 1-5 level of decompositions is used. One key point in DWT is that the original signal can be reconstructed from the divided subimages; this process is called Inverse DWT (IDWT). Mathematically the DWT and IDWT can be stated as below.

Let the low and high pass filter be defined using Equation (1). A signal, F(n) can be decomposed recursively using Equations (2) and (3), where $\mathrm{J}+1$ is the highest resolution level index and $\mathrm{J} 0$ is the low resolution level index. The coefficients

$$
\begin{aligned}
& H(w)=\sum_{k} h_{k} e^{-j k w} \text { and } G(w)=\sum_{k} g_{k} e^{-j k w} \\
& f_{j-1}^{\text {low }}(k)=\sum_{n} h_{n}-2 k f_{j}(n) \text { and } f_{j-1}^{\text {high }}(k)=\sum_{n} g_{n}-2 k f_{j}(n)
\end{aligned}
$$

where $\mathrm{j}=\mathrm{J}+1, \mathrm{~J}, \ldots, \mathrm{J}_{0}$ and $\mathrm{f}_{\mathrm{J}+1}(\mathrm{k})=\mathrm{F}(\mathrm{f}), \mathrm{k} \in \mathrm{Z}, \mathrm{J}+1$ is the high resolution level index and $\mathrm{J}_{0}$ is the low resolution level index. The coefficients from Equation (3) are called DWT of the signal $F(n)$, where $f_{J_{0}}^{\text {low }}(k)$ is the lowest resolution part of $F(n)$ and are called approximation, while the others are the details of $F(n)$ at various bands of frequencies.

$$
\mathrm{f}_{\mathrm{J}_{0}}^{\text {low }}(\mathrm{k}), \mathrm{f}_{\mathrm{J}_{0}}^{\text {high }}(\mathrm{k}), \mathrm{f}_{\mathrm{J}_{0+1}}^{\text {high }}(\mathrm{k}), \mathrm{f}_{\mathrm{J}}^{\text {high }}(\mathrm{k})
$$

Furthermore, the signal $\mathrm{F}(\mathrm{n})$ can be reconstructed from its DWT coefficients recursively using Equation (4).

$$
\mathrm{f}_{\mathrm{j}}^{\text {low }}(\mathrm{n})=\sum_{\mathrm{k}} \mathrm{h}_{\mathrm{n}-2 \mathrm{k}} \cdot \mathrm{f}_{\mathrm{j}-1}^{\text {low }}(\mathrm{k})+\sum_{\mathrm{k}} \mathrm{g}_{\mathrm{n}-2 \mathrm{k}} \cdot \mathrm{f}_{\mathrm{j}-1}^{\text {high }}(\mathrm{k})
$$

To ensure the IDWT and DWT relationship, the orthogonality condition on the filters $\mathrm{H}(\mathrm{w})$ and $\mathrm{G}(\mathrm{w})$ is enforced (Equation 5).

$$
|\mathrm{H}(\mathrm{w})|^{2}+|\mathrm{G}(\mathrm{w})|^{2}=1
$$

The DWT and IDWT for a two dimensional image $F(m, n)$ can be similarly defined by implementing the one dimensional DWT and IDWT for each dimension $\mathrm{m}$ and $\mathrm{n}$ separately, resulting in a pyramid representation of a signal.

Several types of wavelet filters exist. Some examples include Haar, Daubeschies, Coiflets, Symlets, Morlets, Mexican Hat Meyer and Biorthogonal wavelets. More generally, 
The International Journal of Multimedia \& Its Applications (IJMA) Vol.3, No.4, November 2011

Application of DWT divides an image into four subbands (Fig. 3), which arise from separable applications of vertical and horizontal coefficients. The LH, HL and HH subbands represents detailed features of the images, while LL subband represents the approximation of the image. To obtain the next coarse level, the LL subband can further be decomposed (Fig. 3b), thus resulting in the 2-level wavelet decomposition. The level of decomposition performed is application dependent. The present work considers upto four level of decomposition. The advantages of using wavelets are multifolded. The first is that different sized images at different resolution can be analyzed, the coefficients are small in magnitude and the large coefficients coincide with image edges. The edge coefficients within each subband tend to form spatially connected clusters.

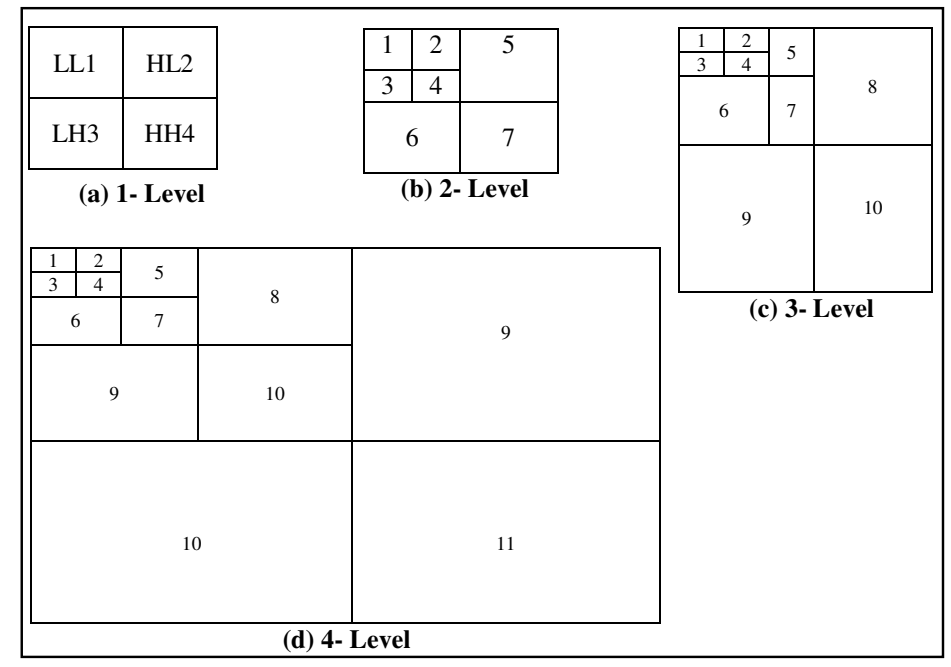

Fig. 3. Wavelet Decompositions

\subsection{Watermarking using Geometric Warping}

The geometric warping method used in this paper follows the methodology proposed by [27] and the readers are referred to this paper for a detailed explanation. A consolidated explanation is presented in this section.

The geometric warping works by embedding the watermark in the relevant and visible part of the video in an imperceptible manner. This approach uses the Peak Signal to Noise Ratio (PSNR) to perform geometric warping and work on the principal that the visible-imperceptible approach can be realized if the watermark is visible according to PSNR definition but imperceptible if someone sees the video. This method changes the border position of the objects as they are untouched by the compression algorithms. To decide for each block, which of the object borders to use during embedding, a Normed Centre of Gravity (NCG) value is used. The NCG value is calculated as follows.

1. Compute the mean values of the rows and columns of the block and store it in $\mathbf{M}_{\mathrm{x}}$ and $\mathrm{M}_{\mathrm{y}}$.

2. Using $\mathrm{M}_{\mathrm{x}}$ and $\mathrm{M}_{\mathrm{y}}$, compute $\mathrm{x}$ - and $\mathrm{y}$-coordinate of the NCV. (This arranges the two vectors in two circles).

3. Compute the 2-D vector $\mathrm{v}_{\mathrm{k}}(\mathrm{k}=\mathrm{x}$ or $\mathrm{y})$ using Equation (1) 


$$
\mathrm{v}_{\mathrm{k}}=\left(\begin{array}{c}
\sum_{i=1}^{\mathrm{n}} \mathrm{m}_{\mathrm{k}}(\mathrm{i}) \cdot \cos \left(\frac{\pi}{\mathrm{n}}+\left((\mathrm{i}-1) \cdot\left(\frac{2 \pi}{\mathrm{n}}\right)\right)\right) \\
\sum_{i=1}^{\mathrm{n}} \mathrm{m}_{\mathrm{k}}(\mathrm{i}) \cdot \sin \left(\frac{\pi}{\mathrm{n}}+\left((\mathrm{i}-1) \cdot\left(\frac{2 \pi}{\mathrm{n}}\right)\right)\right)
\end{array}\right)
$$

4. For each vector, compute the vector angles (Equation 2) and the vector lengths Lx, Ly.

$$
\mathrm{k}=\frac{\mathrm{n} \theta_{\mathrm{k}}}{2 \pi} \quad \text { for }(\mathrm{k}=\mathrm{x} \text { or } \mathrm{y})
$$

The process is shown in Fig. 4 [28].
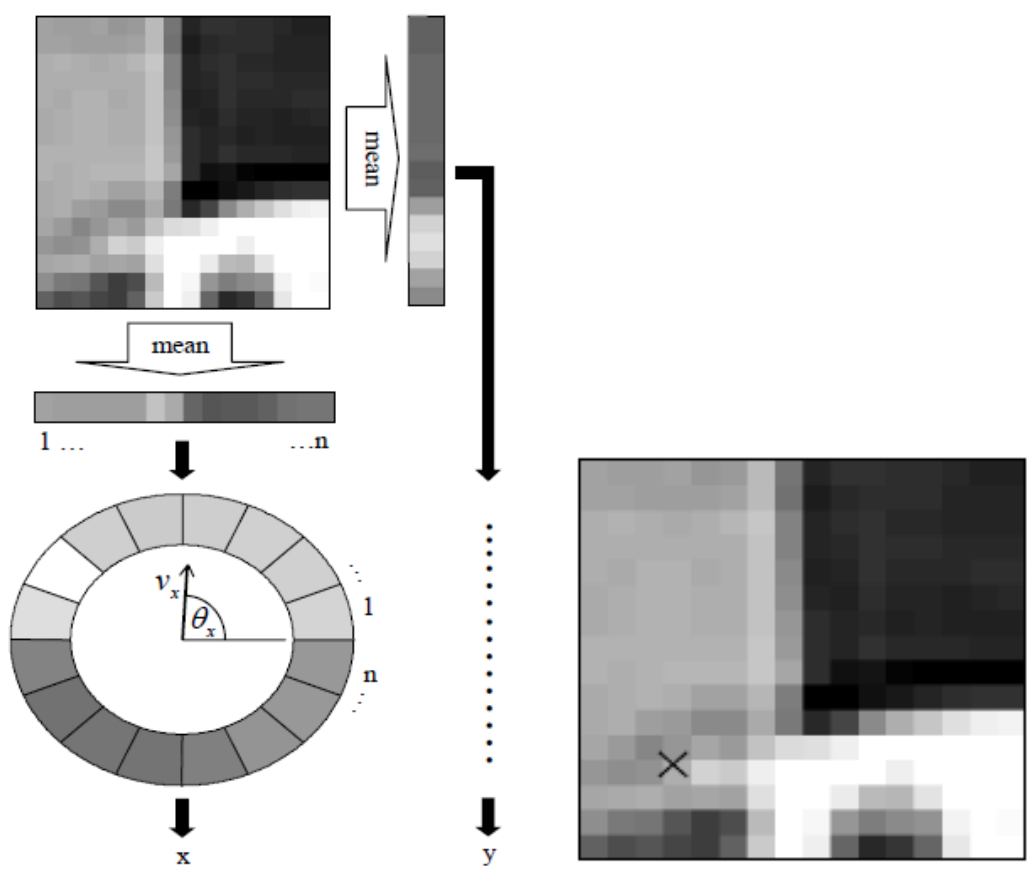

Fig. 4. Computing scheme of the NCG $x, y$-coordinates and NCG $x, y$-coordinates (cross) of the example block

The main advantage of NCG is that it is robust again any compression algorithms. The NCG of blocks with distinct object borders are robust to compression algorithms while NCG blocks without distinct object borders are not robust. Distinct object borders result in high vector lengths, using which the robustness of the NCG $\mathrm{x}, \mathrm{y}$-coordinates can be predicted. The NCG $\mathrm{x}, \mathrm{y}-$ coordinates distortion decreases with increase in vector length. Through experiments, it was proved that the distortion for vector lengths greater than 430 is lower than 0.25 pixels for compression rates upto QP less than equal to 40 . Hence, a watermark can be embedded by quantizing the values of the $\mathrm{x}, \mathrm{y}$ - coordinates of blocks with $\mathrm{L}>430 \mathrm{~b}$ using a quantization step size of one. This watermark will be robust to compression rates upto QP less than equal to 40.

During embedding of watermark, the first step is to find the vector length $\mathrm{L}_{\min }$ which ensures the required robustness. After which, the blocks with $\mathrm{L}>\mathrm{L}_{\min }$ are chosen. To embed on watermark 
The International Journal of Multimedia \& Its Applications (IJMA) Vol.3, No.4, November 2011

bit, the values of the $x, y$ - coordinates of the NCG are quantized. The quantization is done by geometric warping of the block. The direction and strength of warping depends on the original and the quantized NCG-coordinates. To prevent block artifacts to neighbor blocks, the warping process uses different weights for the strength of warping the single pixels. The weighting is computed by using a quadratic function (Fig. 5)

This geometric warping process is used to change the NCG x,y- coordinates.
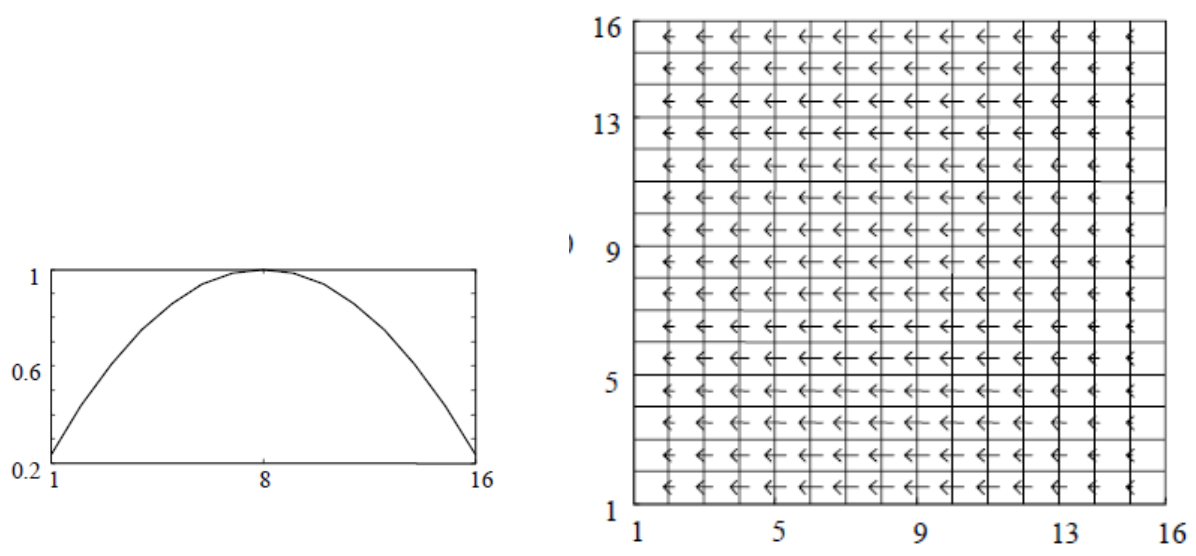

Fig. 5. Weighting function of the pixels and example for geometric warping of the NCG xcoordinate

Author names are to be written in 13 pt. Times New Roman format, centred and followed by a 12pt. paragraph spacing. If necessary, use superscripts to link individual authors with institutions as shown above. Author affiliations are to be written in 12 pt. Times New Roman, centred, with email addresses, in $10 \mathrm{pt}$. Courier New, on the line following. The last email address will have an 18 pt. (paragraph) spacing following.

\section{Proposed WATERMARKING SCHEME}

The proposed watermarking algorithm consists of two stages. The first stage is the embedding stage and the second is extraction of the copyright. The process is shown in Fig. 6 and Fig. 2. 
The International Journal of Multimedia \& Its Applications (IJMA) Vol.3, No.4, November 2011

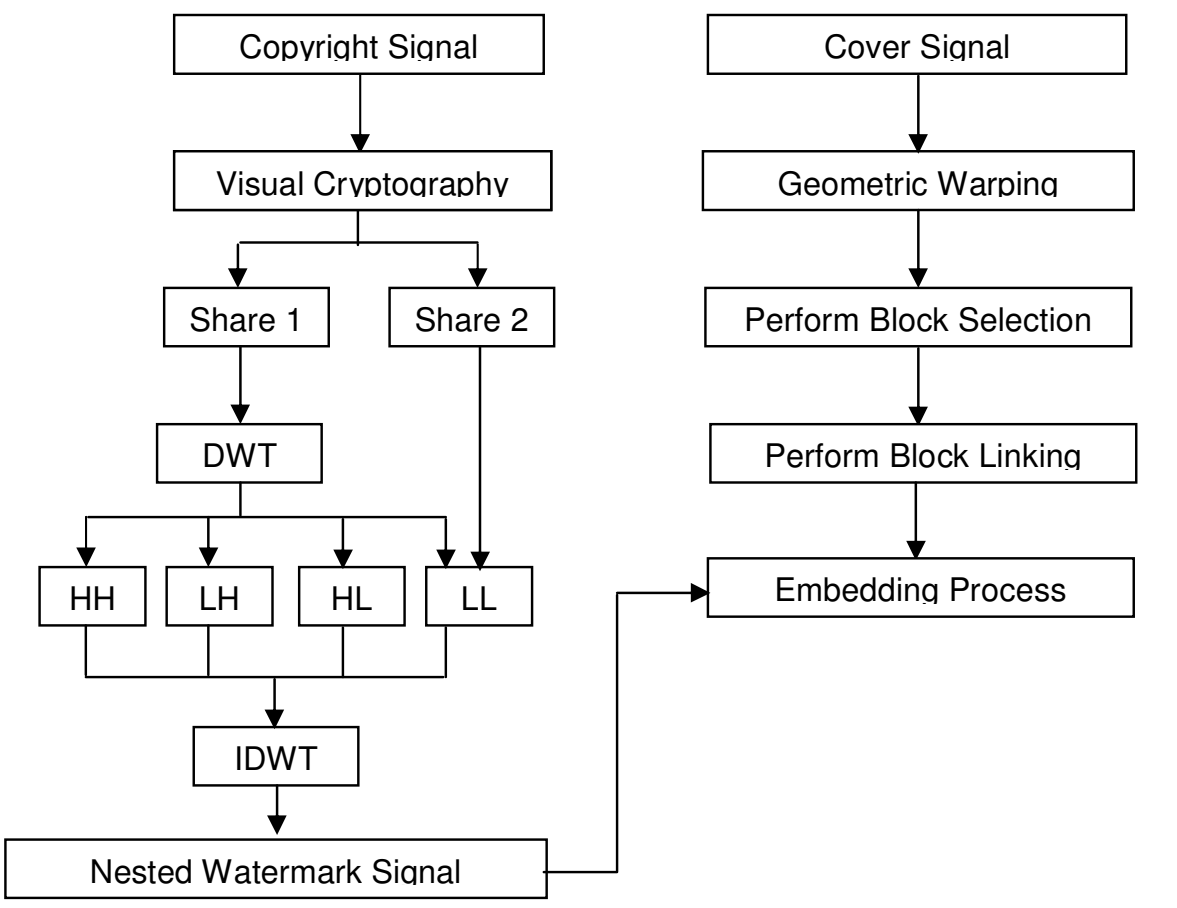

Fig. 6. Embedding Process

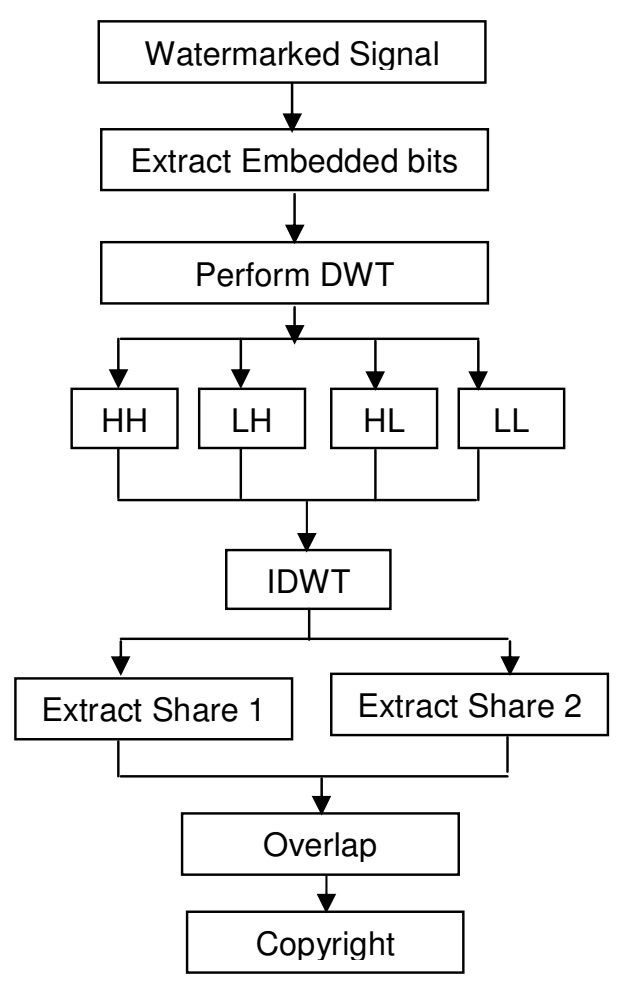

Fig. 7. Watermark Extraction Process 
The International Journal of Multimedia \& Its Applications (IJMA) Vol.3, No.4, November 2011

\subsection{Embedding Process}

The first step is to create nested watermark and the procedure is given in Fig. 8.

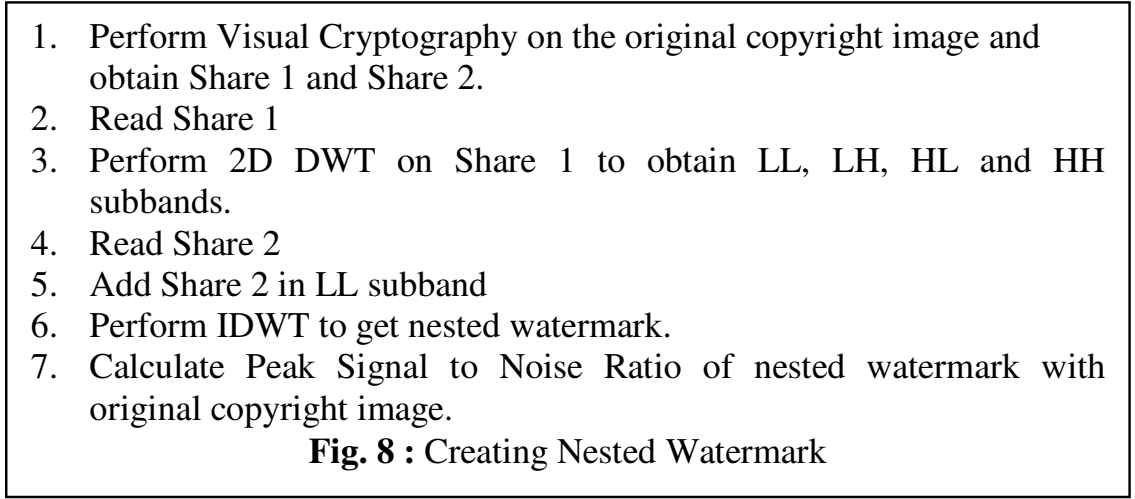

The second step is the watermarking process using geometric warping. To avoid blocking artifacts the geometric warping method uses a block selection procedure. In block selection technique, the selected blocks should maintain minimum distance $d$ to each other in order to prevent artifacts and flickering effects. The minimum distance can be calculated using Equation (3)

$$
\left[\mathrm{d}_{\mathrm{i}}=\left\lfloor\log \left(\mathrm{d} .\left(\mathrm{i}_{\text {size }}-1\right)+1\right)+0, .5\right\rfloor \quad \mathrm{ik} 0 \mathrm{k}=\mathrm{ki}-\mathrm{ii}\right.
$$

where $\mathrm{d}=$ distance and $\mathrm{i}=$ dimensions $(\mathrm{x}, \mathrm{y}$ or $\mathrm{t})$.

The outline of the block selection algorithm is given as follows:

(i) The block group is chosen which is of arbitrary forms.

(ii) The minimum distance $\mathrm{dx}$, dy and dt is computed for each dimension $\mathrm{x}, \mathrm{y}$ and $\mathrm{t}$ of the block group.

(iii) The numbers of blocks inside an ellipsoid are counted for each block. The semi-axes of the ellipsoids are defined by $\mathrm{dx}$, dy and $\mathrm{dt}$. The current block defines the center of the current ellipsoid.

(iv) The blocks with the least neighbors are chosen for watermarking. If more than one chosen block is inside an ellipsoid, only one of them (the first) is used for watermarking. This process is unambiguous.

(v) The chosen blocks and all blocks within the ellipsoids around the chosen blocks will not be considered in the next steps.

(vi) The steps 3-4 are repeated until all blocks are chosen for watermarking or have a distance lower than the minimum to a chosen block.

The blocks chosen using the above algorithm are used for watermark embedding. The watermarking information is embedded by moving the $\mathrm{x}, \mathrm{y}$-coordinates on the quantization lattice to minima/maxima. This is done by geometric warping.

\subsection{Extraction Process}

To extract the watermark bits, the blocks with $\mathrm{L}>\mathrm{L}_{\min }$ are chosen. From the NCG coordinates of the chosen blocks the embedded bit values can be computed. Thus, to extract the watermark, the 
The International Journal of Multimedia \& Its Applications (IJMA) Vol.3, No.4, November 2011

original video is not needed. The NCG is used to detect the watermarked blocks and compute the embedded watermark bit. After successful extraction of the watermarked bits, the next step is to extract the nested watermark. The procedure used is given in Fig. 9.

1. Identify blocking containing watermark bits and extract them to get the nested watermark

2. Apply 2D-DWT to obtain LL, LH, HL and HH subbands

3. Extract Share1 and Share 2 parts

4. Overlap to obtain the copyright image.

Fig. 9. Extraction Process

\section{EXPERIMENTAL RESULTS}

Several experiments were conducted to evaluate the performance of the proposed system. During experimentation several samples were used which included standard test videos and commercially-available movies. The results observed had similar pattern. The results projected in this paper are based on a video clip having 1526 frames (352 × 289 frame size). The experiments were conducted on a Pentium IV with $512 \mathrm{MB}$ memory. The attacks taken into consideration are frame dropping, frame averaging and compression. After extracting and refining the watermarks, a similarity measure, called Normalized Correlation (NC) is used (Equation 4) which gives the cross-correlation, normalized by the reference watermark energy giving unity as the peak correlation.

$$
\mathrm{NC}=\frac{\sum_{\mathrm{i}} \sum_{\mathrm{j}} \mathrm{W}_{\mathrm{ij}} * \mathrm{RW}_{\mathrm{ij}}}{\sum_{\mathrm{i}} \sum_{\mathrm{j}} \mathrm{W}_{\mathrm{i}}{ }_{\mathrm{j}}}
$$

where $\mathrm{W}_{\mathrm{ij}}$ is the original watermark and $\mathrm{RW}_{\mathrm{ij}}$ is the extracted watermark.

Number of frames dropped is a common attack encountered by video watermarking. The reason behind this is the large amount of redundancies that exists between the frames. The effect of this attack is projected in Fig. 10 when tested with different percentage of frames dropped. From the results, it could be seen that the proposed watermarking scheme is better with this type of attack.

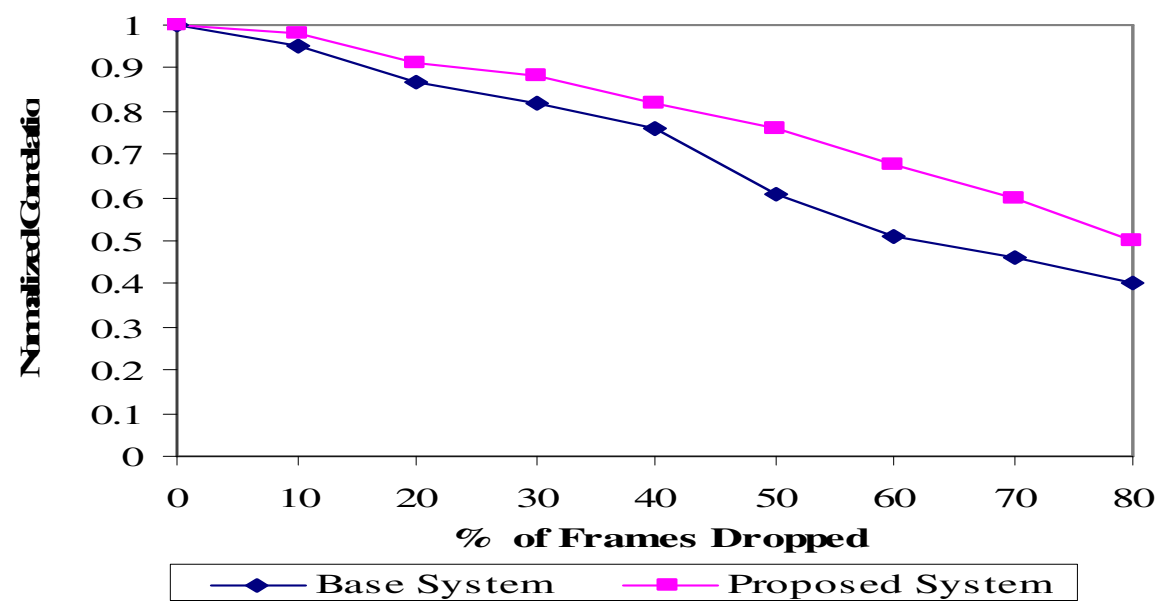

Fig. 10. Number of Frames Dropped 
The International Journal of Multimedia \& Its Applications (IJMA) Vol.3, No.4, November 2011

Frame averaging and statistical analysis is another common attack to the video watermark. When attackers collect a number of watermarked frames, they can estimate the watermark by statistical averaging and remove it from the watermarked video. Fig. 11 shows the results obtained while taking the frame averaging attack into consideration. The results show that fact that the proposed scheme can resist statistical averaging quite well than the base model.

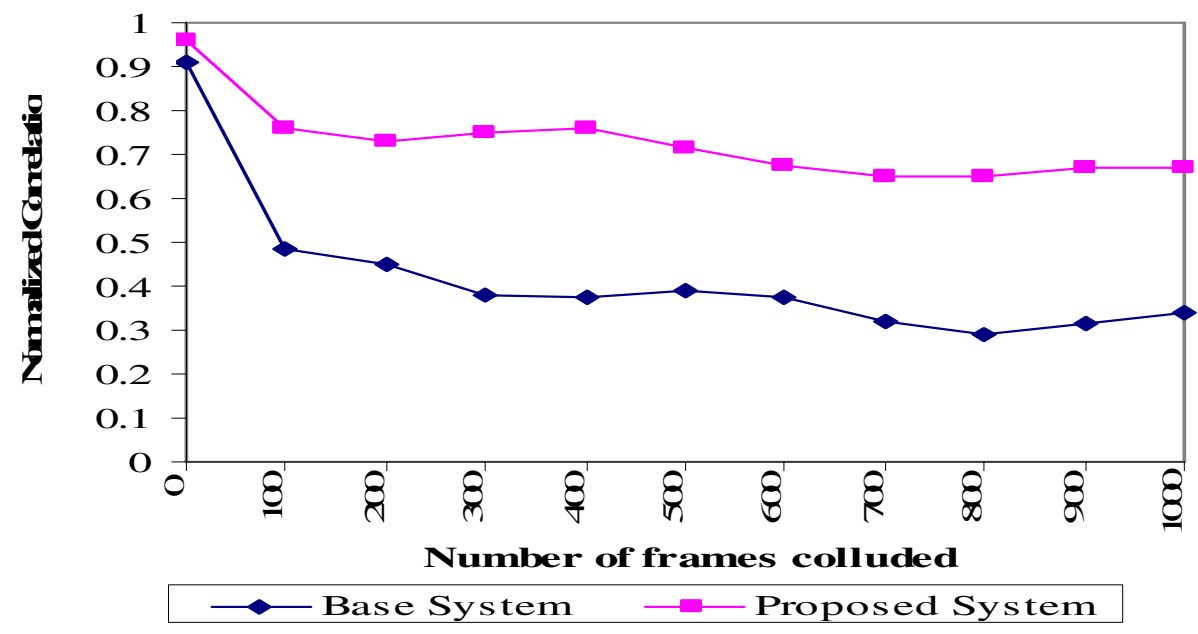

Fig. 11 : Frame Averaging

The NC values of the extracted watermarks with different quality factors are shown in Fig. 12. From the experiment, it is noted that the proposed scheme improves the robustness for watermark protection. It can further be noted, with high quality factor, the algorithm is better in resistance.

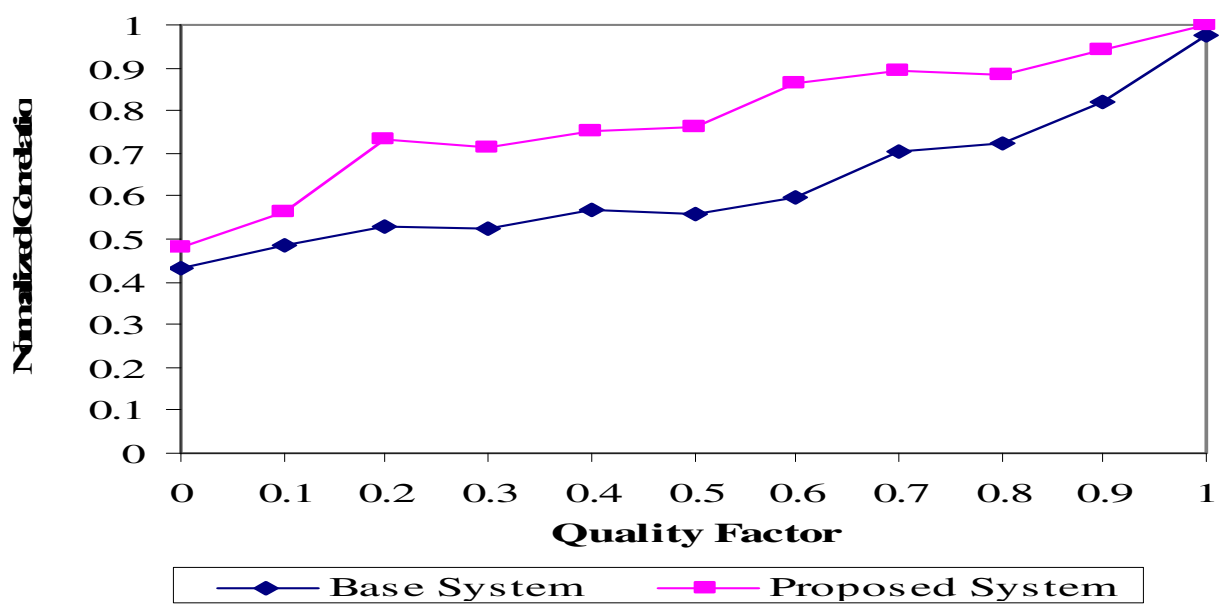

Fig. 12. Compression Attacks

From the various results obtained, it can be concluded that the proposed watermarking scheme is superior to the existing system. 
The International Journal of Multimedia \& Its Applications (IJMA) Vol.3, No.4, November 2011

\section{Conclusions}

This paper proposed a technique for watermarking a video signal with a secret copyright image. The proposed technique is an amalgamation of various techniques like Visual Cryptography, Wavelets and Geometric Warping. Visual cryptography is a concept that is normally used to for hiding multiple watermarks. In this paper, a novel idea of splitting the copyright image into two and creating a nested watermark by storing one split into another is proposed. This method increases the capacity of the watermark. Experimental results proved that the proposed technique is efficient in terms of capacity and robustness against attacks. The present work uses only black and white copyright images, which can be changed to color images and a color visual cryptography method can be combined with this. Moreover, applicability of wavelet packets can be considered for more efficiency.

\section{REFERENCES}

[1] Aggarwal, D., Kaur, S., Anantdeep, E.: An Efficient Watermarking Algorithm to Improve Payload and Robustness without Affecting Image Preceptual Quality, Journal of Computing, vol.2, issue 4 (2010).

[2] Arena, S., Caramma, M.: Digital watermarking applied to MPEG2 coded video sequence exploiting space and frequency masking, Proceedings International Conference on Image Processing (ICIP2000), Vancouver, Canada, vol. 3, pp. 438-441 (2000).

[3] Ateniese, G., Blundo, C., De Santis, A., Stinson, D.R.: Visual cryptography for general access structures, Information Computation, vol. 129, pp.86-106 (1996).

[4] Bonnis, A., Santis, A.: Randomness in secret sharing and visual cryptography schemes, Theor. Comput. Sci, 314, pp 351-374 (2004).

[5] Checcacci, N., Barni, M., Bartolini, F., Basagni, S.: Robust video watermarking for wireless multimedia communications, Proceedings IEEEE Wireless Communications and Networking Confernce 2000, WCNC. 2000, vol. 3, pp. 1530-1535 (2000).

[6] Chun-Shien, L., Shih-Kun, H., Chwen-Jye, S., Mark, L.H. Cocktail watermarking for digital image protection," IEEE Transactions on Multimedia, vol. 2, pp. 209-224 (2004).

[7] Craver, S., Wu, M., Liu, B.: What can we reasonably expect from watermarks?, Proceedings IEEE Workshop on the Applications of Signal Processing to Audio and Acoustics, pp. 223-226 (2001).

[8] Ejima, M., Miyazaki, A.: A wavelet-based watermarking for digital images and video, Proceedings International Conference on Image Processing (ICIP-2000), Vancouver, Canada, vol. 3, pp. 678-681 (2001).

[9] Foo, S., Yeo, T., Huang, D.: An adaptive audio watermarking system, Proceedings IEEE Region 10International Conference on Electrical and Electronic Technology, vol. 2, pp. 509-513 (2001).

[10] Hartung, F., Girod, B.: Watermarking of Uncompressed and Compressed Video, IEEE Transaction Signal Processing, vol.66, no. 3 (Special issue on Watermarking), pp. 283-301 (1998).

[11] Hawkes, L., Yasinsac, A., Cline, C.: An Application of Visual Cryptography to Financial Documents; technical report TR001001, Florida State University (2000).

[12] Houmansadr, A., Ghaemmaghami, S.: A Digital Image Watermarking Scheme Based on Visual Cryptography, International Symposium on Telecommunications, pp. 1-5 (2005). 
The International Journal of Multimedia \& Its Applications (IJMA) Vol.3, No.4, November 2011

[13] Huang, P.S., Chiang, C.S., Chang, C.P., Tu,T.M.: Robust spatial watermarking technique for colour images via direct saturation adjustment, Vision, Image and Signal Processing, IEEE Proceedings, vol. 152, pp. 561-574 (2005).

[14] Kaabneh, K., Youssef, A.: Muteness-based audio watermarking technique, Proceedings International Conference on Distributed Computing Systems Workshop, pp. 379-383 (2001).

[15] Kimpan, S., Lasakul, A., Chitwong, S.: Variable block size based adaptive watermarking in spatial domain, IEEE International Symposium on Communications and Information Technology, ISCIT 2004, vol. 1, pp. 374-377 (2004).

[16] Lee, Y.K., Chen, L.H.: High capacity image steganographic model, Vision, Image and Signal Processing, IEEE Proceedings, vol. 147, pp. 288-294 (2000).

[17] Lin, E., Podilchuk, C., Kalker, T., Delp, E.: Streaming Video and Rate Scalable Compression: What Are the Challenges for Watermarking?, Proceedings the SPIE International Conference on Security and Watermarking of Multimedia Contents III, vol. 4314, San Jose, CA, 22-25 (2001).

[18] Lu, C., Huang, S., Sze, C., Liao, H.Y.M.: Cocktail watermarking for digital image protection,” IEEE Transactions on Multimedia, vol. 2, pp. 209-224 (2000a).

[19] Lu, C., Liao, M., Chen, L.: Multipurpose audio watermarking, Proceedings 15th International Conference on Pattern Recognition 2000, vol. 3, pp. 282-285 (2000b).

[20] Lu, W., Lu, H., Chung, F.L.: Robust digital image watermarking based on subsampling, Applied Mathematics and Computation, vol. 181, pp. 886-893 (2006).

[21] Mohammed, A.A., Hussein, J.A.: Efficient Video Watermarking Using Motion Estimation Approach, 2009 Eight IEEE/ACIS International Conference on Computer and Information Science (icis 2009), pp.593-598 (2009).

[22] Naor, M., Shamir, A.: Visual cryptography, Advances in Cryptology - EUROCRYPT '94, A. De Santis, ed., Lecture Notes in Computer Science, vol. 950, pp. 1-12 (1995).

[23] Naor, M., Pinkas, B.: Visual authentication and identification, Advances in Cryptology CRYPTO'97, Lecture Notes in Computer Science, vol. 1294, pp. 322-336 (1997).

[24] Paul, N., Evans, D., Rubin, A., Wallach, D.: Authentication for remote voting, workshop on humancomputer interaction and security systems, Fort Lauderdale, Florida, April (2003).

[25] Piva, A., Bartolini, F., Barni, M.: Managing copyright in open networks, IEEE Transactions on Internet Computing, vol. 6, Issue. 3, pp. 18-26 (2002).

[26] Praun, E., Hoppe, H., Finkelstein, A.: Robust Mesh Watermarking, ACM SIGGRAPH (1999).

[27] Pröfrock D., Schlauweg M., Müller E.: Video Watermarking by Using Geometric Warping Without Visible Artifacts, Proc. of Information Hiding (8thIH 2006), Alexandria,USA, (2006).

[28] Pröfrock, D., Richter, H., Schlauweg M., Müller E.: H.264/AVC video authentication using skipped macroblocks for an erasable watermark. Proc. of Visual Communications and Image Processing VCIP 5960 (2005)

[29] Qui, G., Marziliano, P., Ho A. T. S., He D., Sun Q.: A hybrid watermarking scheme for H.264/AVC video. Proc. of the ICPR 4, 865-868 (2004).

[30] Ren-Junn, H., Chuan-Ho, K., Rong-Chi,C.: Watermark in color image," Proceedings of the first International Symposium on Cyber Worlds, pp. 225-229 (2002). 
The International Journal of Multimedia \& Its Applications (IJMA) Vol.3, No.4, November 2011

[31] Tai, G.C., Chang, L.W. Visual Cryptography for Digital Watermarking in Still Images, Advances in Multimedia Information Processing - PCM 2004, Category - Watermarking I, Lecture Notes in Computer Science, Springer Berlin / Heidelberg, vol. 3332, pp.50-57 (2005).

[32] Verma, B., Jain, S., Agarwal, D.P., Phadikar, A.: A New color image watermarking scheme, Infocomp, Journal of computer science, vol. 5,N.2, pp. 37-42 (2006).

[33] Wang, C., Nie, X., Wan, X., Wan, W.B., Chao, F.: A Blind Video Watermarking Scheme Based on DWT," iih-msp, 2009 Fifth International Conference on Intelligent Information Hiding and Multimedia Signal Processing, pp.434-437 (2009).

[34] Wu, X., Guan, Z.H. A novel digital watermark algorithm based on chaotic maps, Physics Letters A, vol. 365, pp. 403-406 (2007).

[35] Yang, C.: A note on Efficient Color Visual Encryption, vol.18, pp 367- 372 (2002).

\section{Authors}

Mrs. T. Jayamalar , 4 years of teaching experience. She is currently working as assistant professor in Kovai Kalaimagal College of Arts and Science. Her research interest are Image Processing and Networking. She has 4 publications at International level.

Dr.V.Radha, more than 20 years of teaching experience as reader. Area of Specialization: Image processing, Optimization techniques, Voice Recognition and synthesis. She has 20 publications of National and International level journals and conferences
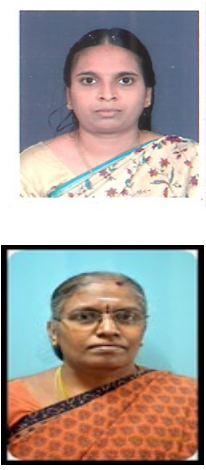